\title{
Research Article \\ Stabilization of Teleoperation Systems with Communication Delays: An IMC Approach
}

\author{
Yuling Li 1 (1) \\ School of Automation and Electrical Engineering, University of Science and Technology Beijing, Beijing 100083, China \\ Correspondence should be addressed to Yuling Li; lyl8ustb@gmail.com
}

Received 9 November 2017; Accepted 17 January 2018; Published 20 February 2018

Academic Editor: Brady King

Copyright ( 2018 Yuling Li. This is an open access article distributed under the Creative Commons Attribution License, which permits unrestricted use, distribution, and reproduction in any medium, provided the original work is properly cited.

\begin{abstract}
The presence of time delays in communication introduces a limitation to the stability of bilateral teleoperation systems. This paper considers internal model control (IMC) design of linear teleoperation system with time delays, and the stability of the closed-loop system is analyzed. It is shown that the stability is guaranteed delay-independently. The passivity assumption for external forces is removed for the proposed design of teleoperation systems. The behavior of the resulting teleoperation system is illustrated by simulations.
\end{abstract}

\section{Introduction}

Teleoperation systems enable humans to extend their capacity to manipulate interfaces with better safety, at less cost, and even with better accuracy. A typical teleoperation system is composed of five parts: a human operator, a master robot which is operated by the human operator, a slave robot, the environment interacting with the slave, and the communication channel between the master and the slave. The main objectives of control design for a bilateral system are the stability of the closed-loop system, position coordination between the master and the slave, and the haptic force display of environment to the human operator.

When there exist significant delays in the communication channel of the teleoperation system, one major issue is the stability of the system [1]. The passivity-based control approaches which are based on scattering theory [1] or wave variable [2] concept are widely used to design stable teleoperation system with time delays. These controllers render the communication link passive and, thus, guarantee stable bilateral teleoperation of any passive environment by any passive user; see [3] and the references therein. For passivitybased controlled teleoperation system, passivity assumption for external forces is required. However, in reality, it is not so easy to satisfy this assumption, and thus this control strategy has its own limitation in real applications.
Realizing the disadvantages in applying passivity-based control schemes in controlling teleoperation systems, we propose an alternative method in which internal model control (IMC) structure is introduced to linear teleoperation system design in this work. IMC was a well-established control strategy around the 1980s and its original configuration and several modified structures have been successfully applied to various applications from chemical processes to automotive systems (see [4-7] and many references therein). The extension to nonlinear systems has also been reported as it shows attractive properties beyond linear system design [8]. Some intelligent methods were introduced to the modification of IMC structures and nonlinear extensions [912]. Moreover, there have been some efforts on extending the IMC design method to nonlinear systems in the linear parameter varying (LPV) framework $[13,14]$ in recent years. The application of IMC in teleoperation systems is also not prior art. Hayn and Schwarzmann have employed IMC structure to design positions controllers for a teleoperation system with a hydraulic manipulator as the slave and a haptic device as the master [15]. However, the authors in [15] assume that no delay exists in the communication. The authors in [16] proposed an IMC design for teleoperation systems with time-varying delays, while, actually, it is a smith-predictorbased design of teleoperation systems. In this work, an IMCbased control structure for delayed teleoperation systems is 
proposed and no restrictive assumptions are made on this structure. The passivity assumption is not required for the proposed control scheme.

The arrangement of the remainder of this paper is as follows. In Section 2 the system modeling and some preliminaries are given. In Section 3, a control architecture is given and its stability is analyzed in Section 4. A simple DOF teleoperation system is given as an example to show the effectiveness of the proposed method in Section 5. Finally, the summary and conclusion of this paper are given in Section 6 .

\section{Preliminaries}

A single-DOF linear master/slave manipulator can be written as [17]

$$
\begin{aligned}
m_{m} \ddot{x}_{m}(t)+b_{m} \dot{x}_{m}(t)+k_{m} x_{m}(t) & =f_{h}(t)+f_{m}(t), \\
m_{s} \ddot{x}_{s}(t)+b_{s} \dot{x}_{s}(t)+k_{s} x_{s}(t) & =-f_{e}(t)+f_{s}(t),
\end{aligned}
$$

where $x_{i}, \dot{x}_{i}, \ddot{x}_{i}$ are the joint positions, velocities, and acceleration values of the master and slave devices with $i=m$ or $s$ representing master or slave robot manipulators, respectively. Similarly, $m_{i}, b_{i}, k_{i}$ are the effective mass, damping, and spring coefficients of the master and slave devices. External forces applied to the devices by the human operator and the environment are represented by $f_{h}, f_{e}$, respectively, while $f_{m}$, $f_{s}$ stand for control signals.

For simplicity, the transfer functions of the master and the slave are given as follows:

$$
\begin{gathered}
G_{m}: G_{m}(s)=\frac{y_{m}(s)}{f_{h}(s)+f_{m}(s)}=\frac{1}{m_{m} s^{2}+b_{m} s+k_{m}}, \\
G_{s}: G_{s}(s)=\frac{y_{s}(s)}{-f_{e}(s)+f_{s}(s)}=\frac{1}{m_{s} s^{2}+b_{s} s+k_{s}} .
\end{gathered}
$$

In this work, the following assumptions are required.

Assumption 1. The forward and backward delays through the communication channel denoted by $T_{1}, T_{2}$, respectively, are assumed to be constant, but of arbitrary value.

Remark 2. Assumption 1 is made for simplicity. The main results in this paper are also valid for teleoperation systems with time-varying delays. In Section 5, we also provide some simulation study for the case with time-varying delays.

\section{IMC Structure for Teleoperation Systems}

This section proposes an IMC-based control structure which guarantees the delay-independent stability of the closed-loop system.

Let us start with the controller design process from one side of the teleoperation system, that is, from the master side, if no confusion arises. Inspired by the traditional IMC structure, we postulate the IMC structure for the master in Figure 1 where $G_{m}$ represents the master dynamics as in (2) and $\widetilde{G}_{m}$ represents the model of the master manipulator. Note that the two-degree-of-freedom IMC structure [4] is utilized since the dynamic characteristics of the two inputs $f_{h}, r_{s d}$ are substantially different. To describe the transparency, the reference signal $r_{s d}$ should be the signals from the slave. Considering the communication delay between the master and the slave, the reference $r_{s d}$ is delayed; that is,

$$
r_{s d}(t)=r_{s}\left(t-T_{2}\right) \text {. }
$$

Obviously, the controllers $C_{11}, C_{12}$ are an operator of $r_{m}, r_{s d}$, respectively; that is, $C_{11}(\cdot)=C_{11}\left(r_{m}(t)\right)$ and $C_{12}=$ $C_{12}\left(r_{s d}(t)\right)$. The human operator $f_{h}(t)$ seems to be a kind of "disturbance" from the original IMC design interpretation; however, we may find that it should not be canceled in our design. The detailed discussion will be given later.

Analogically, the IMC structure for the slave side is depicted in Figure 2, where $C_{21}, C_{22}$ are linear operators of $r_{s}$ and $r_{m d}$; that is, $C_{21}(\cdot):=C_{21}\left(r_{m d}(t)\right)$ and $C_{22}(\cdot):=C_{22}\left(r_{s}(t)\right)$, and

$$
r_{m d}(t)=r_{m}\left(t-T_{1}\right)
$$

The coordinating torques are given as

$$
\begin{aligned}
f_{m}(t) & =C_{12}\left(r_{s}\left(t-T_{2}\right)\right)-C_{11}\left(r_{m}(t)\right), \\
f_{s}(t) & =C_{21}\left(r_{m}\left(t-T_{1}\right)\right)-C_{22}\left(r_{s}(t)\right) .
\end{aligned}
$$

Equations (6) can also be represented by their $S$-functions:

$$
\begin{aligned}
& F_{m}(s)=\left[\begin{array}{ll}
-C_{11}(s) & C_{12}(s) e^{-s T_{2}}
\end{array}\right]\left[\begin{array}{c}
R_{m}(s) \\
R_{s}(s)
\end{array}\right], \\
& F_{s}(s)=\left[\begin{array}{ll}
C_{21}(s) e^{-s T_{1}} & -C_{22}(s)
\end{array}\right]\left[\begin{array}{c}
R_{m}(s) \\
R_{s}(s)
\end{array}\right] .
\end{aligned}
$$

The control architecture is shown in Figure 3.

Remark 3. From Figure 3 and compared with the classical IMC structure [4], we may find that the external forces $f_{h}, f_{e}$ are the "disturbances" according to the classical IMC interpretation. The difference is that these "disturbances" act on the master and the slave directly. In reality, these forces are kind of "excitation signals" and should not be canceled.

Remark 4. Actually, the output $y_{m}, y_{s}$ can be positions or velocities, or even the states of the master and the slave. In this paper, we assume that only the position is available for measurement; that is, $y_{m}=x_{m}$ and $y_{s}=x_{s}$.

\section{Stability Analysis}

In this section, the stability of the closed-loop system is discussed.

Suppose $\mathbf{r}(t):=\left[\begin{array}{c}r_{m}(t) \\ r_{s}(t)\end{array}\right], \mathbf{u}(t):=\left[\begin{array}{l}f_{m}(t) \\ f_{s}(t)\end{array}\right], \mathbf{y}_{o}(t):=\left[\begin{array}{c}y_{m}(t) \\ y_{s}(t)\end{array}\right]$, and $\mathbf{w}(t):=\left[\begin{array}{c}f_{h}(t) \\ -f_{e}(t)\end{array}\right]$; then the closed-loop system in Figure 3 can be redrawn as in Figure 4 where $\mathbf{G}(s)=\left[\begin{array}{cc}G_{m}(s) & 0 \\ 0 & G_{s}(s)\end{array}\right]$ and $\widetilde{\mathbf{G}}(s)=\left[\begin{array}{cc}\widetilde{G}_{m}(s) & 0 \\ 0 & \widetilde{G}_{s}(s)\end{array}\right]$. 


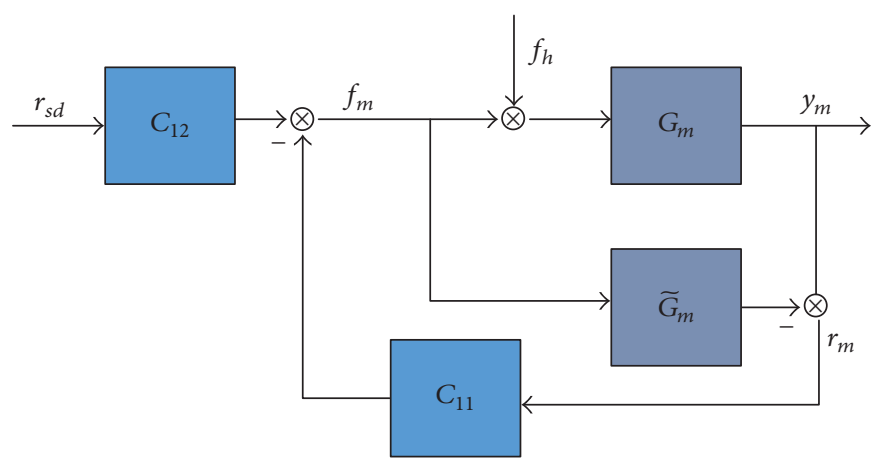

FIGURE 1: IMC structure of the master subsystem.

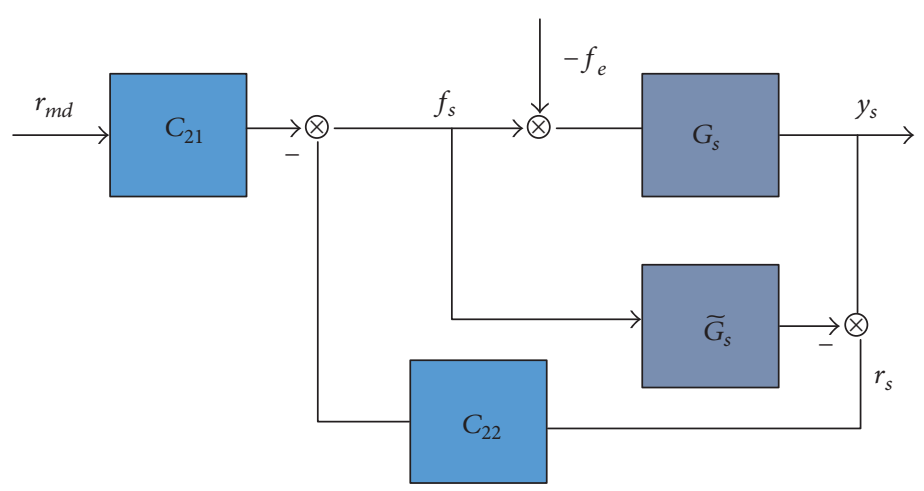

FIGURE 2: IMC structure of the slave subsystem.

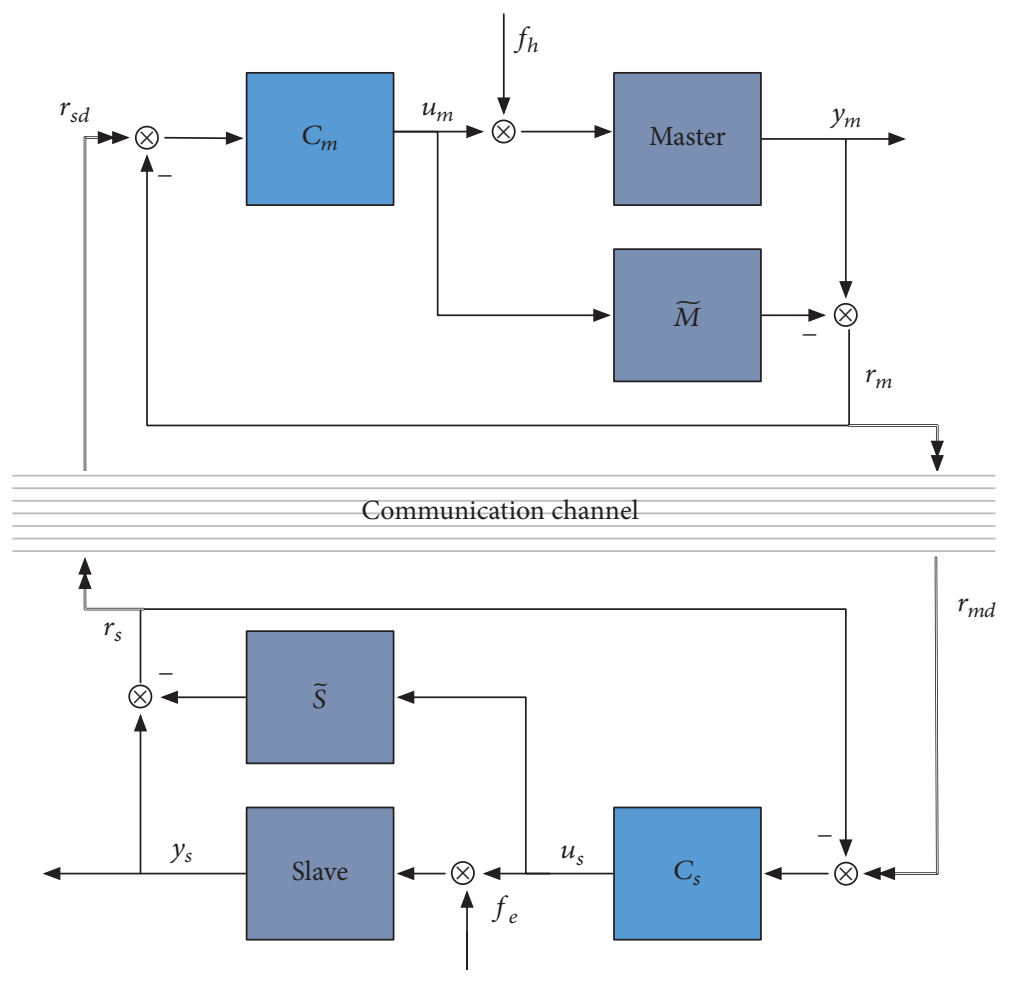

FIGURE 3: IMC structure of teleoperation systems. 


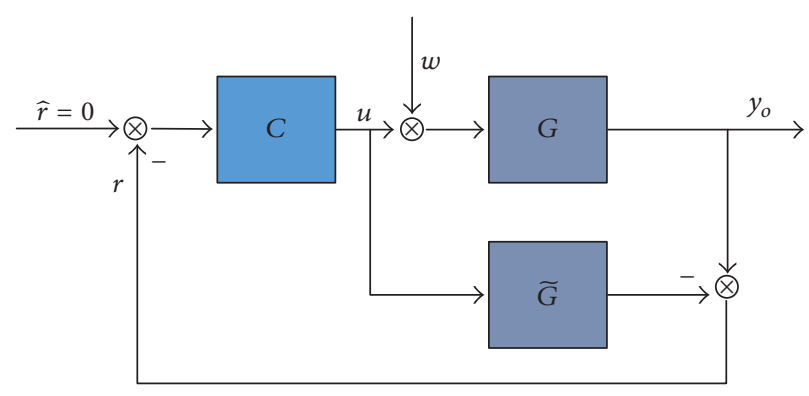

FIGURE 4: Equivalent IMC structure of teleoperation systems.

Let $\mathbf{R}, \mathbf{U}$ be the Laplace transform of $\mathbf{r}, \mathbf{u}$; then the controllers (7) can be rewritten as

$$
\mathbf{U}(s)=-\mathbf{C}(s) \mathbf{R}(s),
$$

where

$$
\mathbf{C}(s)=\left[\begin{array}{cc}
C_{11}(s) & -C_{12}(s) e^{-s T_{2}} \\
-C_{21} e^{-s T_{1}} & C_{22}(s)
\end{array}\right]
$$

From the block diagram of the IMC structure shown in Figure 4, the output $\mathbf{y}_{o}$ is related to the input $\mathbf{w}$ as

$$
\mathbf{Y}_{o}(s)=\mathbf{G}\left(\mathbf{I}+\mathbf{C}(\mathbf{I}-\widetilde{\mathbf{G}} \mathbf{C})^{-1} \mathbf{G}\right)^{-1} \mathbf{W}(s) \text {. }
$$
as

Since $\mathbf{C}(\mathbf{I}-\widetilde{\mathbf{G}} \mathbf{C})^{-1}=(\mathbf{I}-\mathbf{C} \widetilde{\mathbf{G}})^{-1} \mathbf{C},(10)$ can be rewritten

$$
\mathbf{Y}_{o}(s)=\mathbf{G}(\mathbf{I}-\mathbf{C} \widetilde{\mathbf{G}}+\mathbf{C G})^{-1}(\mathbf{I}-\mathbf{C} \widetilde{\mathbf{G}}) \mathbf{W}(s) .
$$

When the system model $\widetilde{\mathbf{G}}$ matches the plant $\mathbf{G}$ perfectly, that is, $\widetilde{\mathbf{G}}=\mathbf{G}$, one has

$$
\mathbf{Y}_{o}(s)=\mathbf{G}(\mathbf{I}-\mathbf{C G}) \mathbf{W}(s) .
$$

It can be seen that the internal stability is always ensured as long as a stable parameter $\mathbf{C}_{0}(s)=\left[\begin{array}{ll}C_{11}(s) & C_{12}(s) \\ C_{21}(s) & C_{22}(s)\end{array}\right]$ is used to control the stable plant $\mathbf{G}$. Like the traditional IMC system, we have the following important property.

Theorem 5 (dual stability). Assume that the master/slave model and the master/slave manipulator dynamics match perfectly; that is, $\mathbf{G}_{i}(s)=\widetilde{\mathbf{G}}_{i}(i=m, s)$. Then the stability of $\mathbf{C}_{0}$ is sufficient for the stability of the overall closed-loop system.

Remark 6. In the proposed control design, there are no passivity assumptions for human forces $f_{h}$ and environmental forces $f_{e}$. These forces can be any bounded signals from the IMC interpretation.

Remark 7. Actually, when $\mathbf{G}=\widetilde{\mathbf{G}}$, the system is basically open loop. Hence this IMC-based design of teleoperation systems provides the open-loop advantages. When the perfect model is not available, that is, $\mathbf{G} \neq \widetilde{\mathbf{G}}$, the overall system is a closed-loop system. Thus, the IMC control strategy has the advantages of both the open-loop and the closed-loop structures [18]. However, the stability condition for the case that $\mathbf{G} \neq \widetilde{\mathbf{G}}$ becomes complex, since the subdiagonal delays exist in $\mathbf{C}$, and this will be a subject of ongoing research.

Remark 8. For the case $\mathbf{G}=\widetilde{\mathbf{G}}$, the benefit of this structure is that the communication time delays do not enter into the feedback channel. Hence the stability of the overall system can be simplified given the stable controllers $C_{11}(s), C_{12}(s)$, $C_{21}(s)$, and $C_{22}(s)$. In another way, it means that this control structure can guarantee the stability of the overall system with the communication delays varying from 0 to arbitrary value. This implies a way to choose suitable $C_{11}(s), C_{12}(s), C_{21}(s)$, and $C_{22}(s)$ with sound performance.

Remark 9. Theorem 5 is also applicable to teleoperation systems with time-varying delays since the communication delays do not enter into the feedback loop.

\section{Simulation and Results}

We consider a simple single-DOF teleoperation system with the dynamics (1) with $m_{m}=0.3 \mathrm{~kg}, m_{s}=1 \mathrm{~kg}, b_{m}=1 \mathrm{Ns} / \mathrm{m}$, $b_{s}=3 \mathrm{Ns} / \mathrm{m}, k_{m}=10 \mathrm{~N} / \mathrm{m}$, and $k_{s}=10 \mathrm{~N} / \mathrm{m}$.

The operator is assumed to be with the following dynamics:

$$
f_{h}(t)=f(t)-b_{\mathrm{op}} \dot{x}_{m}(t)-k_{\mathrm{op}} x_{m}(t)
$$

where $f(t)$ is a rectangle signal depicted in Figure $5, b_{\text {op }}=3$, and $k_{\mathrm{op}}=200$. The environmental force is chosen as $f_{e}(t)=$ $-k_{\text {env }} x_{s}(t)\left(k_{\text {env }}=400\right)$.

We first implement the control in Figure 3 assuming that there are no delays in communication channel and obtain the simulation results shown in Figures 7 and 8. The chosen control parameters $C_{i j}$ are represented by their step responses, which are depicted in Figure 6. It can be seen that the master and the slave respond stably. As shown in Figure 7, the slave's motion follows the master's quickly with no delay; however, there is a bit of oscillation in the position of the master, which means the control parameters $C_{i j}$ could be better chosen. Even though the positions between the masters and the slaves achieve perfect tracking, there is static error between the human force and the environmental force (Figure 8). This error may not be canceled since the position 


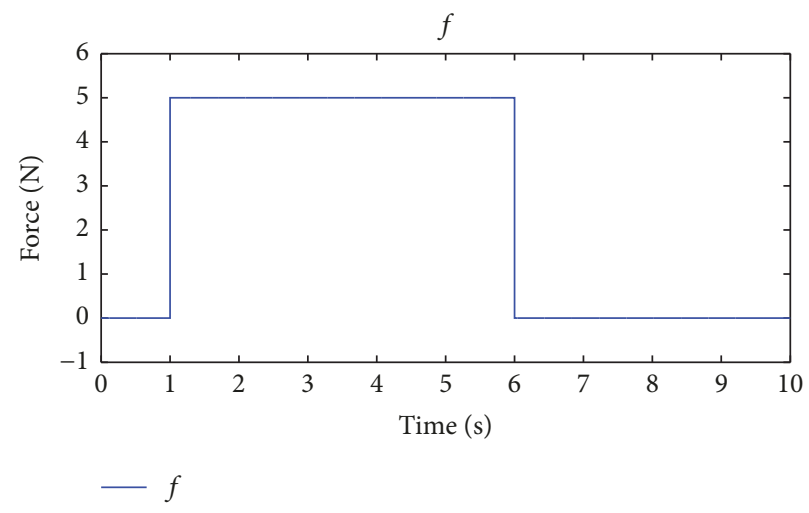

Figure 5: External force $f(t)$.
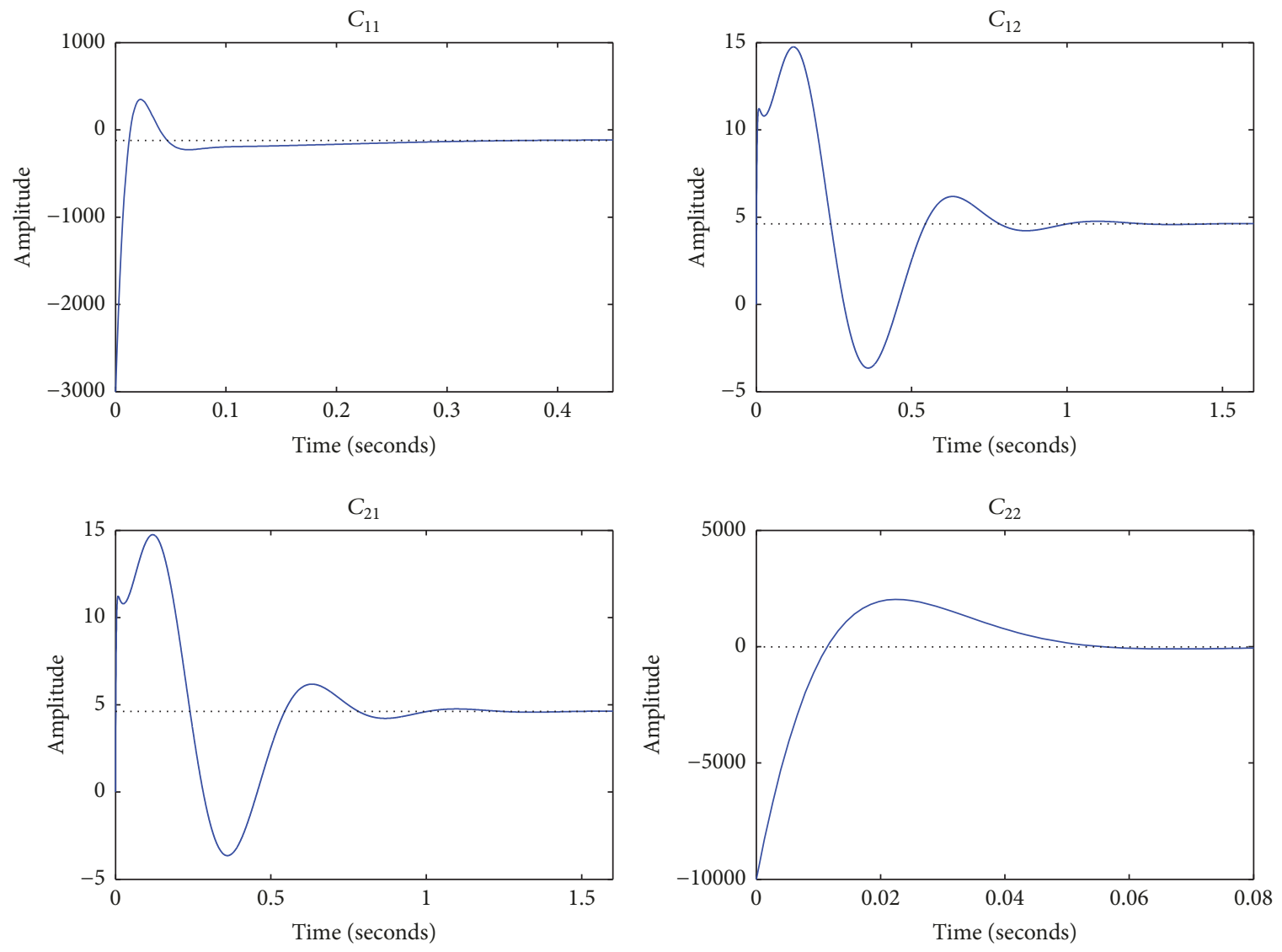

FIgURE 6: Step responses of $C_{11}(s), C_{12}(s), C_{21}(s)$, and $C_{22}(s)$.

tracking and the force tracking are two objectives which require trade-offs.

Now we make simulations for the case when there exist delays in the communication channel. Let us firstly assume that the time delays in the forward channel and backward channel are symmetric; that is, $T_{1}=T_{2}=1 \mathrm{~s}$. The system performance with the designed controller under this case is depicted in Figures 9 and 10. It can be seen from Figure 10 that when the operator exerted the force to the master around $t=1 \mathrm{~s}$, the slave contacted the environment after the delay of 1 second and then received an active force $f_{e}$. The (delayed) position tracking performance depicted in Figure 9 is similar to the one when there are no delays in the communication channel.

We furthermore make simulations for the system with sudden appearing communication delays and sudden disappearing communication delays (both around $t=4 \mathrm{~s}$ ), but the delays are symmetric. For simplicity, we only provide 


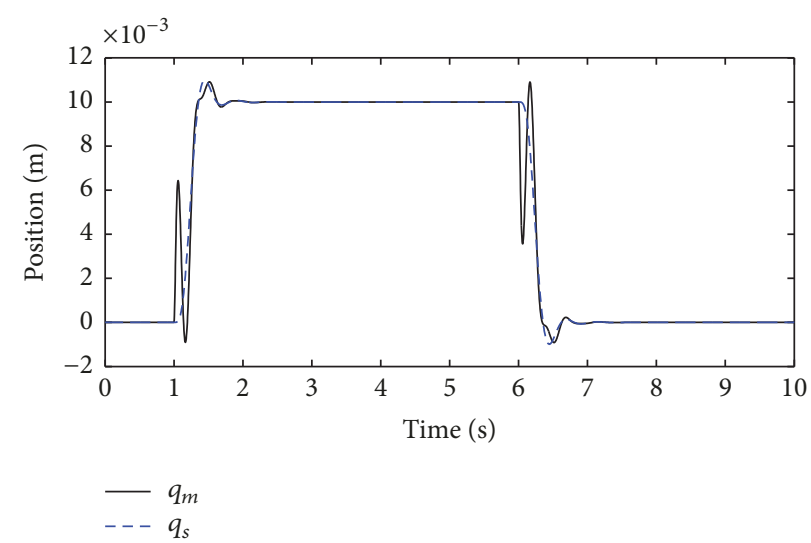

FIGURE 7: Position tracking performance when there are no delays in the communication channel.

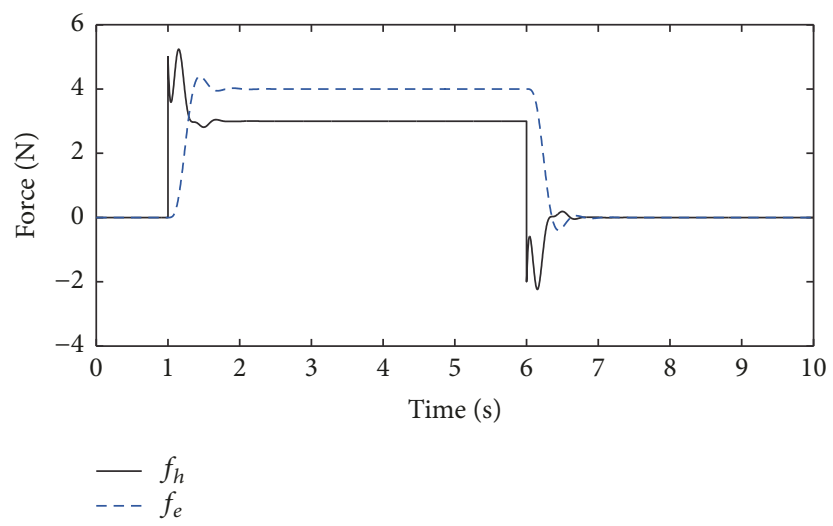

FIGURE 8: Force tracking performance when there are no delays in the communication channel.

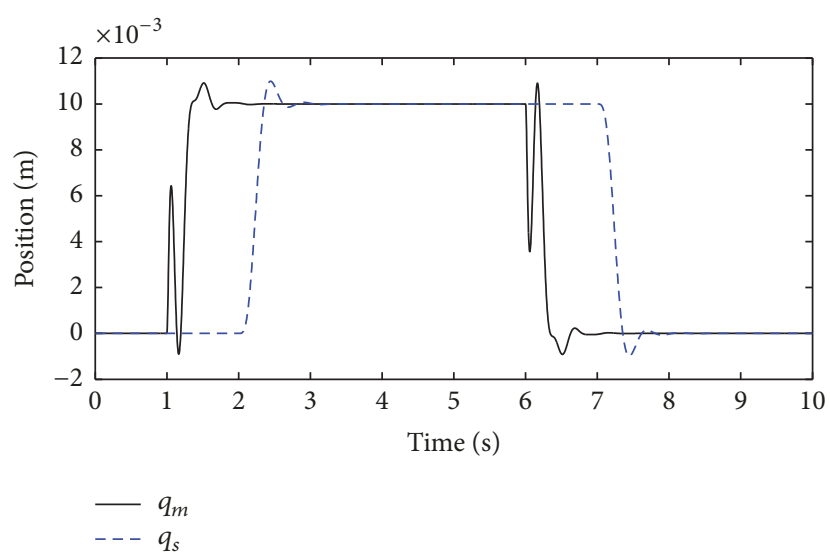

FIgURE 9: Position tracking performance $\left(T_{1}=T_{2}=1 \mathrm{~s}\right)$.

the position tracking performances which are depicted in Figures 11 and 12. It is easy to see that the closed-loop system stays stable in this case. For the time interval with communication delays, the slave follows the master's motion after corresponding delays.
We further concern ourselves with the system's performance when the forward and backward communication delays are not the same. We decrease the forward communication delay to $0.5 \mathrm{~s}$ and increase the backward communication delay to $2 \mathrm{~s}$ and implement the control in Figure 3, 


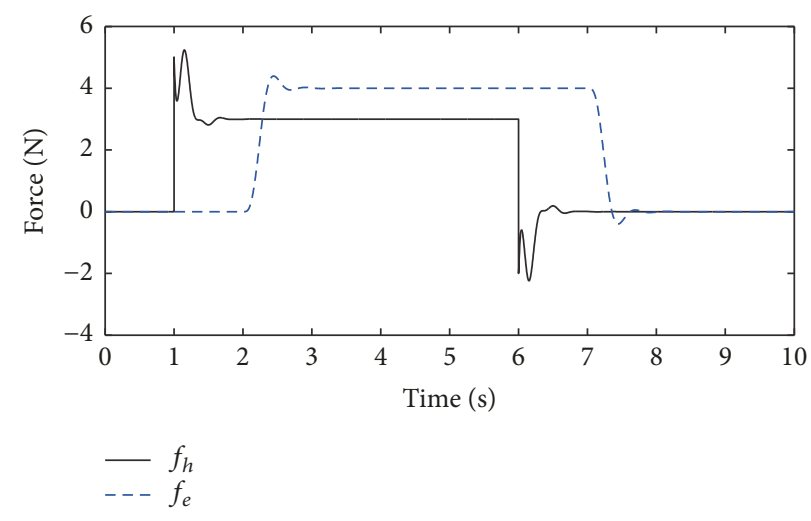

FIgURE 10: Force tracking performance $\left(T_{1}=T_{2}=1 \mathrm{~s}\right)$.

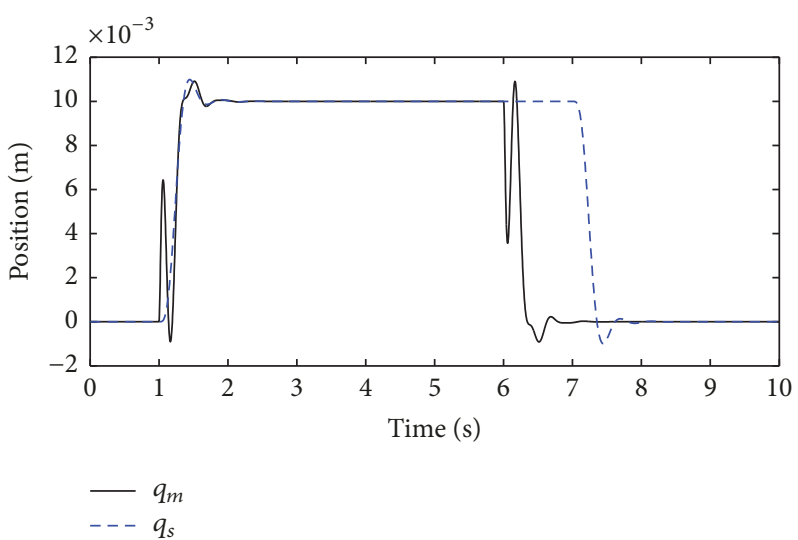

FIGURE 11: Position tracking performance with sudden appearing delays.

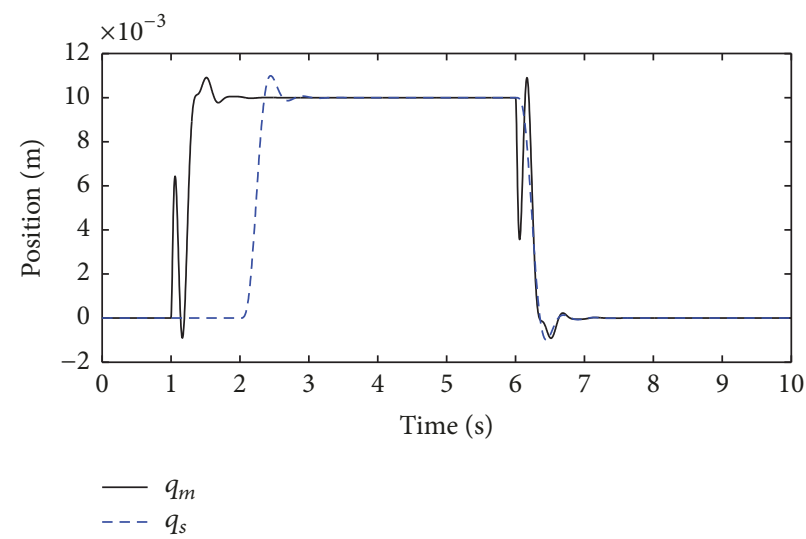

Figure 12: Position tracking performance with sudden disappearing delays.

and then we obtain the simulation results as in Figures 13 and 14. These figures implied that the closed-loop system's stability is guaranteed with unsymmetrical communication delays. Hence, it showed that the designed controller is a delay-dependent stable controller.

Finally, we make simulations for the teleoperation system with varying communication delays. The forward and backward time delays in the communication channel are modeled as $T_{i}(t)=\left|X_{i}(t)\right|(i=1,2)$, where $X_{i}$ are random variables with normal distribution characterized by its mean $\tau_{v}$ and standard deviation $\delta$, denoted by standard notation $X_{i}(\cdot) \sim$ $N\left(\tau_{v}, \delta^{2}\right)$. The forward and backward time delays in the communication channel are chosen with $X_{i} \sim N(0.4,0.01)$. The position tracking performance and force tracking performance are depicted in Figures 15 and 16. It is shown that the system is stable with good tracking performance even 


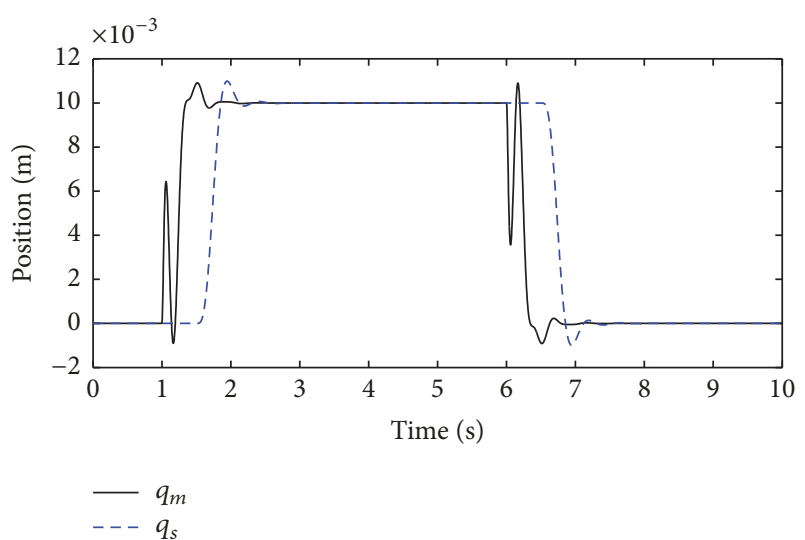

FIGURE 13: Position tracking performance $\left(T_{1}=0.5 \mathrm{~s}, T_{2}=2 \mathrm{~s}\right)$.

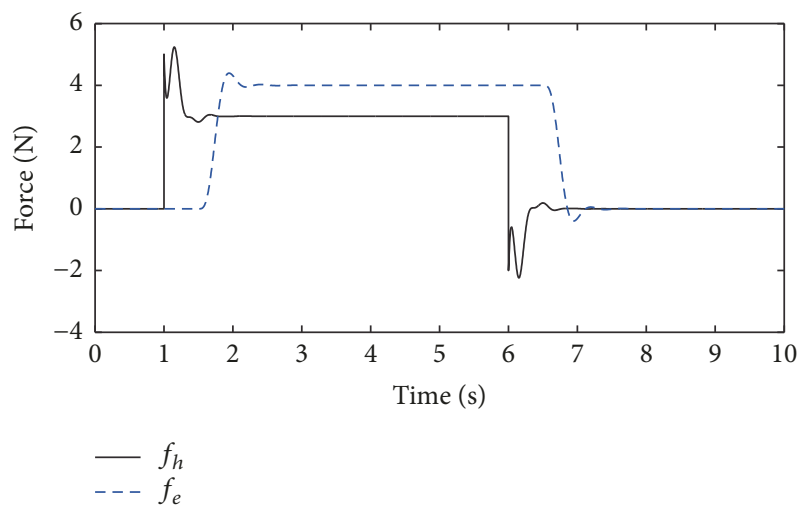

FIGURE 14: Force tracking performance $\left(T_{1}=0.5 \mathrm{~s}, T_{2}=2 \mathrm{~s}\right)$.

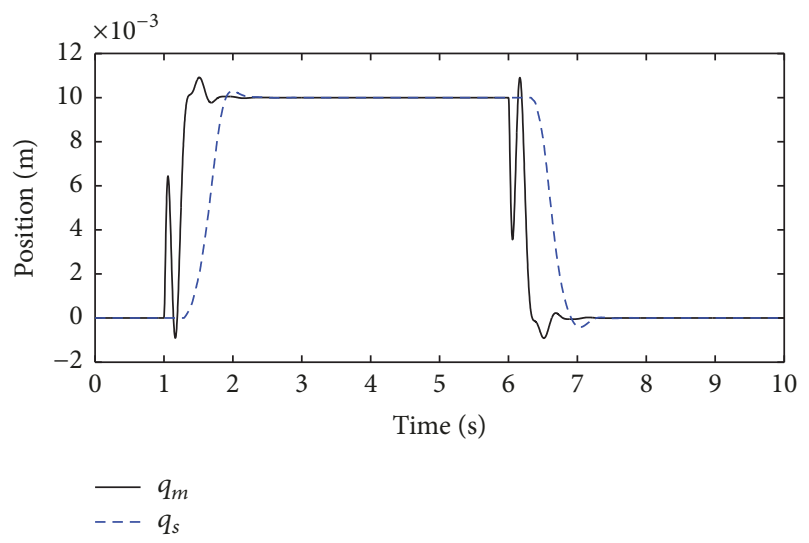

FIGURE 15: Position tracking performance $\left(T_{i}(t)=\left|X_{i}(t)\right|(i=\right.$ $\left.1,2), X_{i} \sim N(0.4,0.01)\right)$.

with bounded stochastic communication delays. This means that our method is also valid for teleoperation systems with varying delays.

\section{Conclusion}

In this paper, we investigate the IMC-based control design of linear teleoperation system with communication delays.

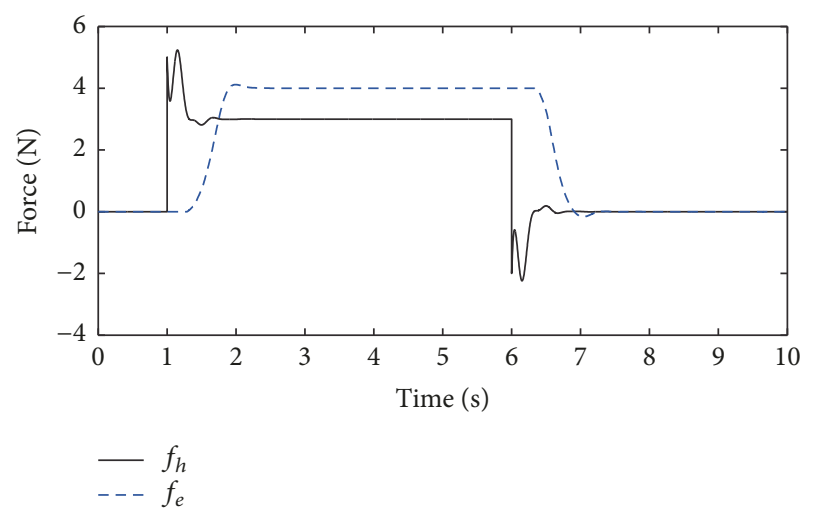

FIGURE 16: Force tracking performance $\left(T_{i}(t)=\left|X_{i}(t)\right|(i=\right.$ $\left.1,2), X_{i} \sim N(0.4,0.01)\right)$.

The stability of the overall system is guaranteed if the perfect model is available. This method removes the passivity assumption for external forces. Simulations of a singleDOF linear teleoperation system show that the stability is guaranteed when the designed controller is applied. Good tracking performance can be achieved if the parameters $C_{i j}$ are chosen suitably. Extensions to the case when the models and the plants are not perfectly matched and to nonlinear teleoperation systems are under study and the research results will be reported in the near future.

\section{Conflicts of Interest}

The author declares that there are no conflicts of interest regarding the publication of this paper.

\section{Acknowledgments}

This work was jointly supported by the National Natural Science Foundation of China (no. 61333002), the Fundamental Research Funds for the Central Universities (nos. FRF-TP-16024A1 and FPR-BD-16-005A), and the Beijing Key Discipline Development Program (no. XK100080537).

\section{References}

[1] R. J. Anderson and M. W. Spong, "Bilateral control of teleoperators with time delay," Institute of Electrical and Electronics Engineers Transactions on Automatic Control, vol. 34, no. 5, pp. 494-501, 1989.

[2] G. Niemeyer and J.-E. Slotine, "Stable adaptive teleoperation," IEEE Journal of Oceanic Engineering, vol. 16, no. 1, pp. 152-162, 1991.

[3] E. Nuño, L. Basañez, and R. Ortega, "Passivity-based control for bilateral teleoperation: A tutorial," Automatica, vol. 47, no. 3, pp. 485-495, 2011.

[4] M. Morari and E. Zafiriou, Robust process control, Prentice hall, Englewood Cliffs, NJ, USA, 1989.

[5] D. Schwarzmann, Nonlinear Internal Model Control with Automotive Applications, Logos-Verlag, 2008.

[6] D. Kim, D. Lee, and K. C. Veluvolu, "Accommodation of actuator fault using local diagnosis and IMC-PID," International 
Journal of Control, Automation, and Systems, vol. 12, no. 6, pp. 1139-1149, 2014.

[7] T. N. L. Vu, J. Lee, and M. Lee, "Design of multi-loop PID controllers based on the generalized IMC-PID method with Mp criterion," International Journal of Control, Automation, and Systems, vol. 5, no. 2, pp. 212-217, 2007.

[8] C. G. Economou, M. Morari, and B. O. Palsson, "Internal model control: extension to nonlinear system," Industrial \& Engineering Chemistry, Process Design and Development, vol. 25, no. 2, pp. 403-411, 1986.

[9] D. W. C. Hot and Z. Ma, "Multivariable internal model adaptive decoupling controller with neural network for nonlinear plants," in Proceedings of the 1998 American Control Conference, ACC 1998, pp. 532-536, Philadelphia, Pa, USA, June 1998.

[10] K. Hunt and D. Sbarbaro, "Neural networks for nonlinear internal model control," IEE Proceedings Part D: Control Theory and Applications, vol. 138, no. 5, pp. 431-438, 1991.

[11] E. P. Nahas, M. A. Henson, and D. E. Seborg, "Nonlinear internal model control strategy for neural network models," Computers \& Chemical Engineering, vol. 16, no. 12, pp. 10391057, 1992.

[12] I. Rivals and L. Personnaz, "Nonlinear internal model control using neural networks: application to processes with delay and design issues," IEEE Transactions on Neural Networks and Learning Systems, vol. 11, no. 1, pp. 80-90, 2000.

[13] W. Xie and T. Eisaka, "Generalized internal model architecture for gain-scheduled control," IMA Journal of Mathematical Control and Information, vol. 25, no. 4, pp. 447-459, 2008.

[14] J. Mohammadpour, J. Sun, A. Karnik, and M. Jankovic, "Internal model control design for linear parameter varying systems," in Proceedings of the 1st American Control Conference (ACC '13), pp. 2409-2414, Washington, DC, USA, June 2013.

[15] H. Hayn and D. Schwarzmann, "Control concept for a hydraulic mobile machine using a haptic operating device," in Proceedings of the 2nd International Conferences on Advances in ComputerHuman Interactions, ACHI 2009, pp. 348-353, Mexico, February 2009.

[16] P. Jian and Q.-Q. Li, "Internal model control for force-reflecting teleoperation systems with varying time-delay," Kongzhi Lilun Yu Yingyong/Control Theory and Applications, vol. 24, no. 1, pp. 13-18, 2007.

[17] P. F. Hokayem and M. W. Spong, "Bilateral teleoperation: An historical survey," Automatica, vol. 42, no. 12, pp. 2035-2057, 2006.

[18] I. Kaya, "IMC based automatic tuning method for PID controllers in a Smith predictor configuration," Computers \& Chemical Engineering, vol. 28, no. 3, pp. 281-290, 2004. 


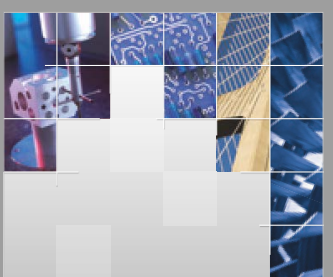

\section{Enfincering}
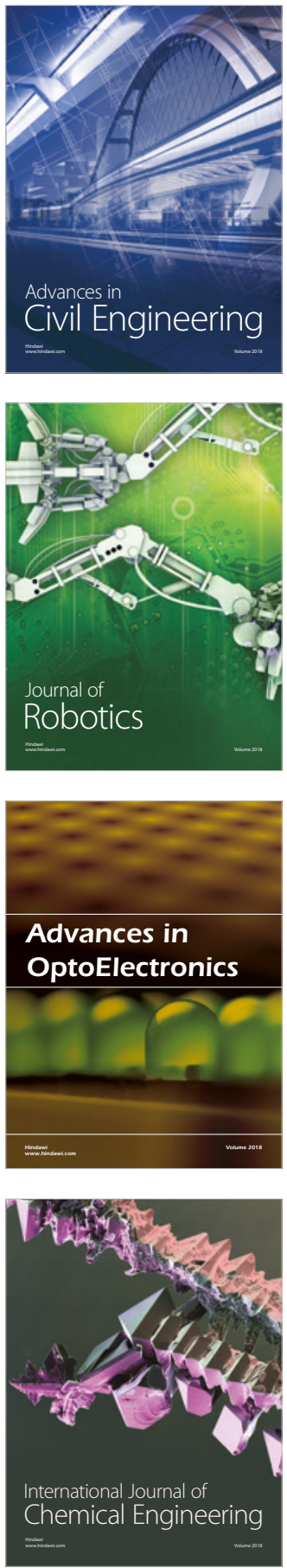

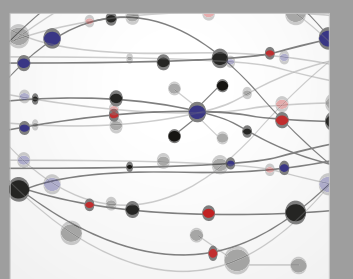

\section{Rotating \\ Machinery}

The Scientific World Journal

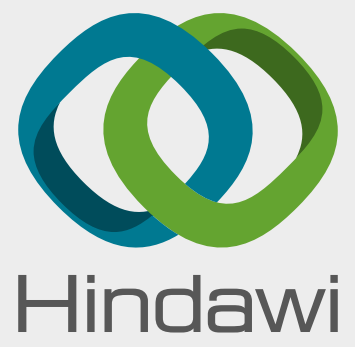

Submit your manuscripts at

www.hindawi.com
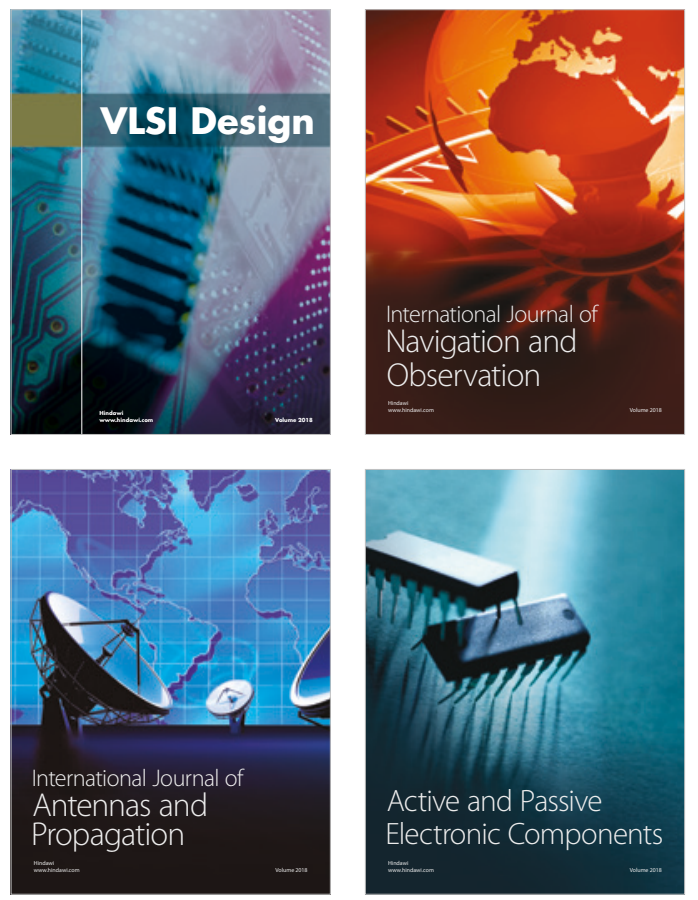
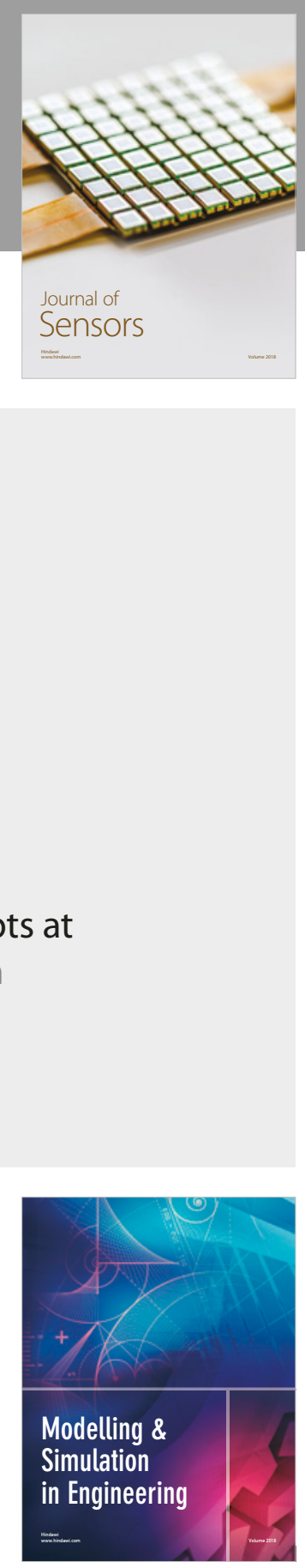

\section{Advances \\ Multimedia}
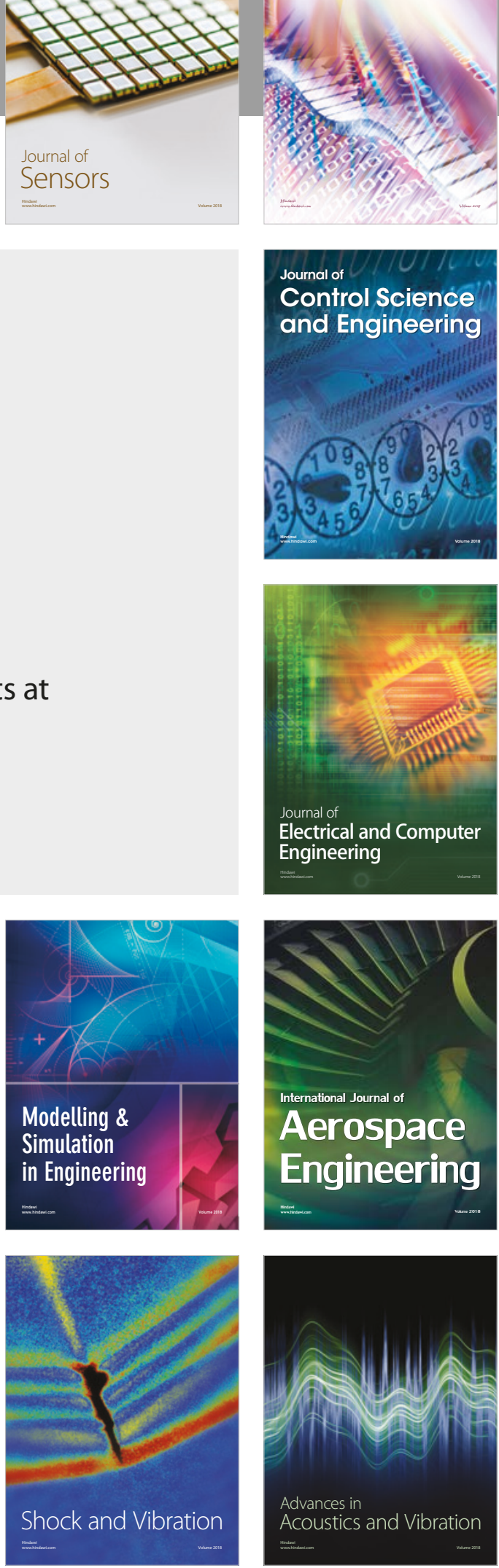